\title{
A study on silicosis among employees of a silica processing factory in the Central Province of Sri Lanka
}

\author{
A D Siribaddana ${ }^{1}$, K Wickramasekera ${ }^{1}$, W M Palipana ${ }^{2}$, M D Peiris ${ }^{3}$, B K A Upul ${ }^{1}$, K P Senevirathna ${ }^{1}$, \\ D L Dassanayake ${ }^{1}$
}

(Index words: silicosis, quartz, crystalline silica, progressive massive fibrosis)

\begin{abstract}
Objectives The silica industry is rapidly expanding in Sri Lanka. The main objective of this study was to assess the prevalence of silicosis among workers exposed to silica dust, and to describe the disease pattern of the affected. We also screened the affected workers for tuberculosis.

Methods Employees of a silica factory $(n=250)$ were screened using a questionnaire. Details of duration of silica exposure and work category within the industry were recorded. All symptomatic workers were investigated with chest radiography and screened for tuberculosis. International Labour Organisation criteria for diagnosis of silicosis were used for diagnosis.

Results Of the 250 employees 25 (10\%) had respiratory symptoms. Chest radiographs of 14 workers (5.6\%) showed abnormalities diagnostic of silicosis. The mean age of the affected workers was 29 years. One worker had radiological changes suggestive of progressive massive fibrosis. Of 14 patients, 13 had duration of exposure less than 10 years suggestive of accelerated silicosis. Among the 14 workers diagnosed with silicosis five $(35.7 \%)$ had Mantoux induration of more than $10 \mathrm{~mm}$ suggestive of latent tuberculesis.

Conclusions Silica workers at the investigated industrial facility are at a significant risk of developing accelerated silicosis. Prevalence of infection with mycobacteria was high. Education of workers, improvement of protective measures and regular monitoring of the work environment should be undertaken to avert this deadly disease.
\end{abstract}

Ceylon Medical Journal 2016; 61: 6-10

DOI: http://doi.org/10.4038/cmj.v61i1.8252

\section{Introduction}

Silicosis refers to a well-recognised, spectrum of pulmonary diseases caused by inhalation of free crystalline silica (silicon dioxide) [1-4]. The silica industry is rapidly expanding in Sri Lanka because we are a major exporter of silica as powdered quartz which contains more than $95 \%$ of silica. Processed quartz is exported in bulk. Quartz is used for making microchips for computers and electronic industry.

Sri Lanka has large deposits of quartz mainly in the Central Province. Quartz is mined from underground deposits and processed in quartz processing plants mainly in Kandy and Matale districts in the Central Province. Quartz contains more than 95\% silica. During processing, large cubes of quartz are mechanically crushed to produce fine particles with particle size less than 5 microns of aerodynamic diameter. These particles are inhalable and get easily air borne during processing, exposing workers to fine quartz particles.

Inhalation of crystalline silica is associated with many debilitating pulmonary diseases. Three main clinical syndromes have been described, namely chronic, accelerated, and acute silicosis. They are mainly differentiated according to the degree of exposure to silica dust [2]. Chronic and accelerated silicosis have many common radiographic features, and are clinically differentiated on the interval between exposure and the development of symptoms. Accelerated silicosis develops within 10 years of the initial exposure and is associated with high level exposure. Patients with accelerated silicosis are at greater risk for progressive massive fibrosis. Acute silicosis is characterised by rapid onset of symptoms following overwhelming exposure [1-4]. Silicosis is also associated with an increased risk of, mycobacterial

${ }^{1}$ Respiratory Unit, Teaching Hospital Kandy, ${ }^{2}$ Occupational Health Unit, Department of Labour and ${ }^{3}$ Respiratory Unit, District General Hospital Matale, Sri Lanka.

Correspondence: ADS, e-mail: <chamath1122@gmail.com>. Received 18 March and revised version accepted 12 December 2015.

This is an open-access article distributed under the terms of the Creative Commons Attribution License, which permits unrestricted use distribution, and reproduction in any medium, provided the original author and source are credited. 
infection, autoimmune disorders, airflow obstruction and chronic bronchitis [1]. The International Agency for Research in Cancer [IARC] classified silica dust as carcinogenic to humans in 1996 [5].

Sri Lanka does not have well developed programmes for health monitoring of the workforce or environmental monitoring measures. Since there is no proven therapy for silicosis, which has very high morbidity and mortality, efforts should be directed towards prevention through a comprehensive lung health protection programme focused on minimising exposure by improved factory standards and use of personal protective equipment $[3,4]$.

The main objective of the study was to assess the occurrence of silicosis and to describe the disease pattern among workers exposed to silica dust in a silica processing industry in Sri Lanka. We also screened the affected workers for tuberculosis which is an important treatable complication of silicosis.

\section{Methods}

The study was carried out in a silica factory in Matale District, Central Province of Sri Lanka which was established 12 years back. It carries out crushing and grinding of quartz containing stones to powder form. The quarts crushing plant, silica powder weighing and packing plant, the laboratory which analyses the final product to confirm its quality and an administrative block are situated in its premises. There were 250 employees working there at the time of the study.

Approval for this study was granted by the Ethics Committee of Teaching Hospital Kandy. The study was carried out in January 2012. Prior to this study no formal medical examination had been carried out in this factory.

The research team visited the silica factory and all 250 employees were informed about the research. They were educated about respiratory symptoms associated with dust exposure. A screening questionnaire was used to assess symptoms of silicosis as well as tuberculosis which is a recognised complication of silicosis. In addition to demographic data the recorded information included symptoms (cough, shortness of breath, chest tightness, fever, loss of weight, loss of appetite and haemoptysis), duration of work (number of hours per day, number of working days per month and number of employment) and type of work (machine operator, driver, supervisor, packing or laboratory assistant).

Employees who had any of the above symptoms were interviewed individually and were further investigated. The number of symptomatic workers was 25 . They were further investigated with chest radiography, sputum direct smears for acid and alcohol fast bacilli and mycobacterial culture. Mantoux test was performed on all 25 symptomatic workers. We carried out the mantoux test using 2 tuberculin units.
The chest radiographs were reported according to the guidelines of the International Labour Organisation (ILO) and the diagnosis of silicosis was made according to the following criteria as per the guidelines of the ILO [7].

- A history of silica exposure sufficient to cause the degree of illness and the appropriate latency from the time of first exposure

- Chest imaging (usually a conventional chest radiograph) that shows opacities consistent with silicosis.

- Absence of another diagnosis more likely to be responsible for the observed abnormalities.

Data from symptomatic workers were entered in to an Excel sheet and was analysed using SPSS software.

\section{Results}

Out of the 250 total employees, 25 (10\%) had respiratory symptoms. Fourteen (5.6\%) patients were diagnosed as having silicosis according to ILO criteria. There were 12 (85.7\%) males and two (12.5\%) females. The mean age of the affected was 29.9 years (SD 8.9). Chest rediographs of 14 workers (5.6\% of all workers) showed abnormalities diagnostic of silicosis that fulfilled the ILO criteria. The radiological pattern of simple silicosis was observed in 13 affected workers. None of the chest X rays showed egg shell calcification of hilar nodes. One worker had radiographic features suggestive of progressive massive fibrosis (PMF). A high resolution CT scan of the chest confirmed the massive fibrosis and excluded the possibility of an underlying malignancy.

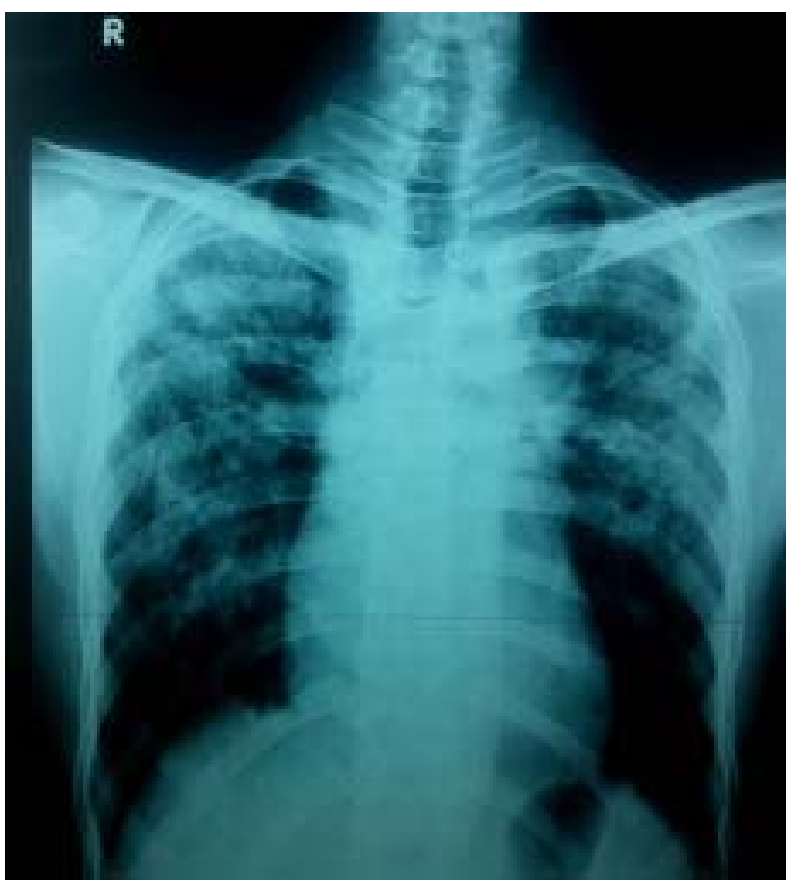

Figure 1. The chest radiograph of the patient with massive pulmonary fibrosis. 


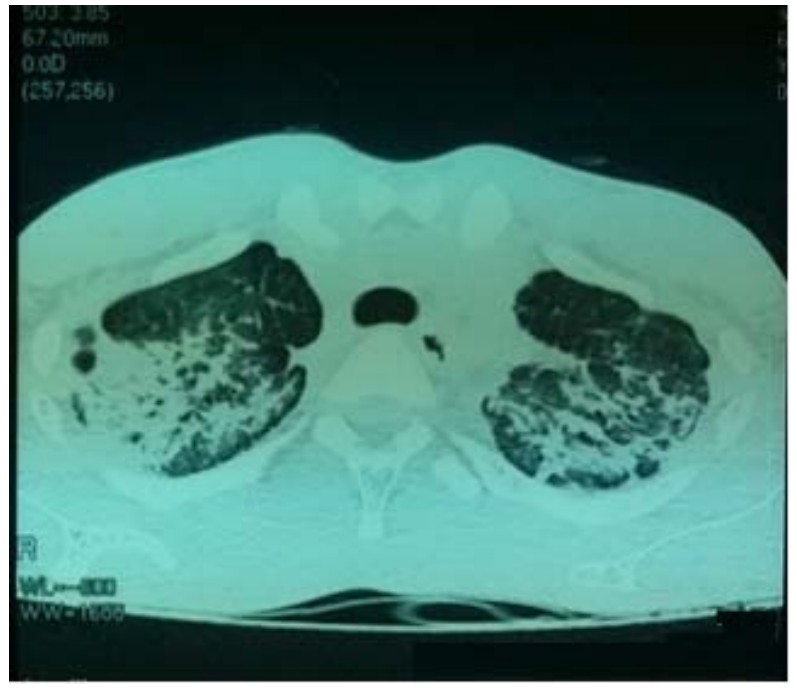

Figure 2. Axial section of upper chest of the CT scan of the patient with progressive massive fibrosis.

Table 1. The distribution of symptoms among patients with silicosis

\begin{tabular}{lc}
\hline Symptom & Number (\%) \\
\hline Shortness of breath on exertion & $14(100)$ \\
Cough & $9(64.3)$ \\
Chest pain & $9(64.3)$ \\
Loss of weight & $6(42.8)$ \\
Loss of appetite & $4(28.5)$ \\
Wheezing & $2(14.3)$ \\
Fever & $2(14.3)$ \\
Haemoptysis & $1(7.1)$ \\
\hline
\end{tabular}

Affected workers had progressive dyspnoea. The patient with PMF was dyspnoec at rest. The employees who had fever and haemoptysis had bacterial chest infections.

We recorded the duration of exposure among workers with silicosis (Table 3). Thirteen (92\%) employees had been exposed to silica dust for less than 10 years, which is compatible with accelerated silicosis. Job description of patients with silicosis were recorded (Table 3).

Table 2. Duration of exposure

\begin{tabular}{lc}
\hline Exposure duration & Number \\
\hline Less than 5 years & 6 \\
5 to 10 years & 7 \\
More than 10 years & 1 \\
Total & 14 \\
\hline
\end{tabular}

Table 3. Type of work of patients with silicosis

\begin{tabular}{lc}
\hline Type of work & Number \\
\hline Machine operator & 8 \\
Quality control officer & 1 \\
Vehicle operator & 1 \\
Weight recorder & 1 \\
Mechanic & 1 \\
Lab assistant & 1 \\
Powder packing worker & 1 \\
Total & 14 \\
\hline
\end{tabular}

In this factory $56 \%$ of employees were machine operators and 8 out of 14 (57.1\%) with silicosis were machine operators. They were involved in crushing silica into fine powder form. All employees worked an average of 8 hours per day and 28 days per month.

On screening of the symptomatic employees for tuberculosis we found that none of the workers were positive for acid fast bacilli on direct smear at the time of investigation. Mycobacterial cultures were negative in all. Among the 14 workers diagnosed of silicosis five (35.7\%) had Mantoux reaction of more than $10 \mathrm{~mm}$ which was indicative of latent tuberculesis infection. The odds ratio for rate of TB infection in workers with silicosis when compared with the symptomatic workers without silicosis was 5.55 (95\% CI 0.4- 152).

Table 4. Results of Mantoux test among the 25 symptomatic workers

\begin{tabular}{lcc}
\hline & With silicosis & $\begin{array}{l}\text { With respiratory } \\
\text { symptoms but no } \\
\text { silicosis }\end{array}$ \\
\hline Mantoux test positive & 5 & 1 \\
Mantoux test negative & 9 & 10 \\
Total & 14 & 11 \\
\hline
\end{tabular}

\section{Discussion}

In this cross sectional descriptive study we found that $5.6 \%$ of the 250 employees were fulfilling the ILO criteria for diagnosis of silicosis. Thirteen out of 14 (92\%) patients had duration of exposure less than 10 years which was compatible with accelerated silicosis. They have a higher chance of developing diffuse fibrosis which may lead to PMF and to respiratory failure [8].

The average age of the affected was 29 years. Since silicosis is a progressive disease which has no cure, the 
young age of the affected is of concern. The employee with PMF was 25 years old and the duration of exposure to silica was only 18 months. These findings alone suggest heavy exposure of workers to respirable silica in the factory we studied. Egg shell calcification of hilar nodes which occurs due to chronic low grade exposure and mainly a feature of chronic silicosis was not seen in any of the affected [4].

Machine operators were the commonest category of workers affected. They are directly involved in crushing the quartz stones to powder form, suggesting heavy exposure to respirable silica. The rest of the affected employees handled the powdered form directly apart from the mechanic who serviced the machines. The laboratory assistant who analysed the final product to certify the quality of the powdered product was affected too. She hardly went out into the crushing plant as the powdered form was brought to her.

Workers with exposure duration of more than 5 years had a $1.6 \%$ risk of having silicosis compared to workers who were exposed for less than 5 years. It is known that duration of exposure is positively correlated with the risk of developing silicosis [3]. Different studies have reported varying incidence of silicosis ranging from $3.2 \%$ in symptomatic individuals to $22 \%$ in postmortem studies $[4,8]$. In our study we found that out of the 25 symptomatic workers 14 were affected, indicating a high incidence of $56 \%$ among the symptomatic group. This finding is more significant as the duration of exposure of affected employees was less than 10 years. This indicates a high degree of exposure. The severe forms of silicosis observed in this industry also indicate high level of exposure to respirable silica. Machine operators and other employees who were directly exposed to dust were more affected compared to the less exposed occupation groups. This suggests inadequate protective measures.

Silicosis is a strong risk factor for development of tuberculosis. A study done in South Africa has shown the risk of tuberculesis to be 13.4 times more in workers with silicosis [9]. In our study we found that none of the patients as positive for acid fast bacilli on direct smear or mycobacterial culture of sputum. This excluded active pulmonary TB at the time of the study. But the workers who had silicosis had a higher rate of positive Mantoux reaction than the workers who did not have silicosis. This indicates that the rate of latent TB infection in this group is higher which increases the chance of developing active TB later in life and emphasises the need for close follow up.

The major drawback of this study is that only the symptomatic workers from the total 250 workforce were investigated. Only the 25 symptomatic employees underwent chest radiography and investigations for tuberculosis. Due to resource constraints the total workforce could not be investigated. Hence the workers with forms of silicosis in which the workers were asymptomatic would have been missed.
This study shows the importance of screening the workers who are exposed to silica and close follow up patients. To the best of our knowledge routine formal screening of employees of silica industry is not carried out in Sri Lanka. We strongly recommend carrying out routine investigation of all workers who are exposed to silica. They need to be kept in surveillance even after cessation of employment and they are no longer exposed to silica, as silicosis with fatal outcomes may develop later in life.

The National Institute for Occupational Safety and Health (NIOSH) in United States has recommended an exposure limit of $0.05 \mathrm{mg} / \mathrm{m}^{3}$ to respirable silica [10]. Environmental monitoring with measurement of respirable silica and dust levels should be done in all factories involved in silica industry. Education of employers and employees of silica industry, mandating health surveillance for workers at risk of developing silicosis and improving industrial environment are essential as this industry is rapidly developing in Sri Lanka.

\section{Acknowledgements}

We acknowledge the staff of respiratory units in Teaching Hospital Kandy and District Hospital Matale, in making this research a success.

\section{Conflicts of interests}

There are no conflicts of interest.

\section{References}

1. Adverse effects of crystalline silica exposure. American Thoracic Society Committee of the Scientific Assembly on Environmental and Occupational Health. Am J Respir Crit Care Med 1997; 155: 761-5.

2. Health effects of occupational exposure to respirable crystalline silica. NIOSH, Publication No. 2002-129. DHHH (NIOSH).

3. Weissman DN, Banks DE. Silicosis in Interstitial Lung Disease, $4^{\text {th }}$ ed, King TE Jr, Schwarz MI (Eds), B.C. Decker, Hamilton, ON, Canada 2003, 387.

4. Merget R, Bauer T, Küpper HU, et al. Health hazards due to the inhalation of amorphous silica. Arch Toxicol 2002; 75: 625 .

5. The international Agency for Research in Cancer. (Scientific Publication no 68). Lyon France, 1996.

6. Leung CC, Yu ITS, Cheng W. Silicosis. Lancet 2012; 379: 2008-18.

7. International Labour Office (ILO) Guidelines for the Use of the ILO International Classification of Radiographs of Pneumoconioses, Revised Edition 2000 (Occupational Safety and Health Series, No. 22). International Labour Office, Geneva, 2002. 
8. Velan G M, Kumar RK, Cohen DD. Pulmonary inflammation and fibrosis following sub acute inhalational exposure to silica. Pathology 1993; 25: 282-90.

9. Eva Hnizdo, Jill Murray Risk of pulmonary tuberculosis relative to silicosis and exposure to silica dust in South African gold miners. Occupation Environ
Med 1998; 55: 496-502.

10. Criteria for a recommended standard: occupational exposure to crystalline silica. HEW Publication NIOSH; US Department of Health, Education and Welfare, Public Health Service, Center for Disease Control, National Institute for Occupational Safety and Health, 1974. 